\title{
Rotating linear polarization imaging technique for anisotropic tissues
}

Ran Liao

Nan Zeng

Tsinghua University

Graduate School at Shenzhen

Laboratory of Optical Imaging and Sensing

Shenzhen 518055 China

\author{
Xiaoyu Jiang \\ Dongzhi Li \\ Tianliang Yun \\ Tsinghua University \\ Graduate School at Shenzhen \\ Laboratory of Optical Imaging and Sensing \\ Shenzhen 518055 China \\ and \\ Tsinghua University \\ Key Laboratory for Molecular and Nanosciences \\ of Education Ministry \\ Department of Physics \\ Beijing 100084 China
}

\section{Yonghong He}

Tsinghua University

Graduate School at Shenzhen

Laboratory of Optical Imaging and Sensing

Shenzhen 518055 China

\section{Hui Ma}

Tsinghua University

Graduate School at Shenzhen

Laboratory of Optical Imaging and Sensing

Shenzhen 518055 China

and

Tsinghua University

Key Laboratory for Molecular and Nanosciences of Education Ministry

Department of Physics

Beijing 100084 China

\begin{abstract}
A novel rotating linear polarization imaging technique is developed to characterize the anisotropic properties of tissues. Differences of orthogonal linear polarization with different incident and detection polarization angles are fitted to an analytical function to retrieve a set of parameters. Experiments with different tissues and Monte Carlo simulations indicate that two of the parameters, $G$ and $\varphi_{3} / 2$, are correlated to the anisotropic property and the orientation angle of the fibrous structure in the media. The technique can be used for clinical diagnosis. (๑) 2010 Society of Photo-Optical Instrumentation Engineers. [DOI: 10.1117/1.3442730]
\end{abstract}

Keywords: scattering; polarization; imaging; tissue; anisotropy.

Paper 09573R received Dec. 24, 2009; revised manuscript received Feb. 2, 2010; accepted for publication Apr. 8, 2010; published online Jun. 9, 2010.

\section{Introduction}

There has been growing interest in studies of polarization imaging in turbid media for achieving higher imaging quality or better characterization of the samples. ${ }^{1-4}$ The primary advantage for clinical application of polarization imaging is that it suppresses backscattered photons from deep tissues and improves the image contrast and resolution in the superficial layers where many cancers start to develop. Degree of polarization (DOP) imaging with linearly polarized light has been successfully used in clinical diagnosis of many cancers. ${ }^{2}$ Other polarization measurements, such as Mueller matrix polarimetry ${ }^{5,6}$ and Stokes polarimetry, ${ }^{7}$ have also been used to retrieve structural and optical information of turbid media.

Address all correspondence to: Hui Ma, Laboratory of Optical Imaging and Sensing, Graduate School at Shenzhen, Tsinghua University, Shenzhen 518055 China. Tel/Fax: 86-755-26036238; E-mail: mahui@tsinghua.edu.cn
Most biomedical tissues are anisotropic, and many contain prominent fibrous structures. ${ }^{8}$ It has been found in our work and by other authors that linear polarization images with single incident polarization can be very different when the incident polarization or sample orientation is changed. ${ }^{7}$ It could be uncertain whether the observed differences represent physiological differences or the optical artifacts due to changes in incident polarization or sample orientations for different tests. ${ }^{9-11}$ Such uncertainty may affect objective diagnostics in clinical applications. Objective and quantitative characterization of anisotropic tissues remains a challenge.

In this work, we present a novel technique, rotating linear polarization imaging (RLPI), which provides a set of parameters to objectively and quantitatively characterize the anisotropic properties of the scattering media. It is based on a reflective linear polarization imaging system but with

1083-3668/2010/15(3)/036014/6/\$25.00 @ 2010 SPIE 

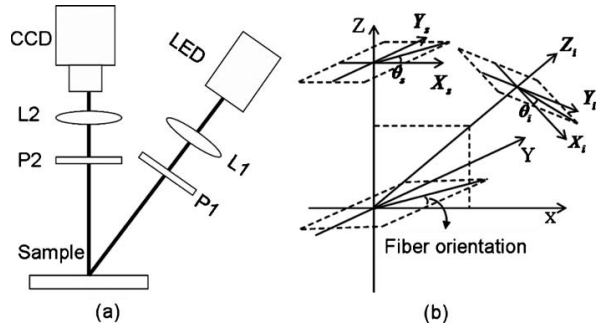

(b)

Fig. 1 (a) Schematics of experimental setup and (b) reference frames of experiment. $\theta_{i}$ and $\theta_{s}$ represent the incidence and detection polarization angles.

independently rotating incident and detection linear polarization angles. Linear differential polarization (LDP) images are fitted at each pixel to an analytical function of the incident and detection polarization angles to retrieve a set of new images of the fitting parameters. Both experiments on different tissues and Monte Carlo simulations show that at least two of the parameters have clear physics meanings, representing the structure anisotropy and the orientation angle of the fibrous structure in the samples. The images are all independent of the incident and detection polarization angles.

\section{Experiment Setup and Data Processing}

The experimental setup (Fig. 1) is similar to a typical reflective linear polarization imaging system. ${ }^{2} 650$-nm light from a 1 -W LED is collimated by a lens L1, propagates though a linear polarizer $\mathrm{P} 1$, and illuminates the sample at $25 \mathrm{deg}$ to the normal of sample surface, which is defined as the $\mathrm{Z}$ axis. Backscattered photons from the sample pass through the analyzer P2, are collected by lens L2, and recorded by a 12-bit charge-coupled device (CCD) (Retiga EXi, QImaging, Surrey, British Columbia, Canada). The image size is $520 \times 696$ pixels and each pixel is $13 \times 13 \mu \mathrm{m}^{2}$. Driven by two-step motors, both P1 and P2 can rotate around their optical axis to vary the polarization angles for illumination $\theta_{i}$ and for detection $\theta_{s}$. The samples are different types of tissues, such as muscles, connective tissues, and fats from different animals, including bovine, chicken, and porcine. A glass plate is placed in close contact to the sample to direct surface glare away from the detector.
In each experiment, we record a series of images $I\left(\theta_{i}, \theta_{s}\right)$. For each incident polarization angle $\theta_{i}$, we take a number of image pairs corresponding to orthogonal detection polarization angles $\theta_{s}$ and $\theta_{s}+\pi / 2$. However, at least once we choose $\theta_{s}=\theta_{i}$ to obtain the DOP image. Then we calculate LDP at each pixel $(x, y)$ :

$$
\operatorname{LDP}\left(\theta_{i}, \theta_{s}\right)=I\left(\theta_{i}, \theta_{s}\right)-I\left(\theta_{i}, \theta_{s}+\pi / 2\right) .
$$

For a fixed $\theta_{s}$, the $\operatorname{LDP}_{x, y}\left(\theta_{i}\right)$ at each pixel $(x, y)$ is cosinoidal [Fig. 2(a)]:

$$
\mathrm{LDP}_{x, y} /\left(I_{\text {in }} / 2\right)=h_{1} \cos \left(2 \theta_{i}-h_{2}\right)+h_{3},
$$

where $I_{\text {in }}$ is the incident intensity.

It was found in the experiments that Eq. (2) stays valid for all the different samples we have tested. In fact, a similar cosinoidal expression was also proposed for polarization measurements in transmission mode. ${ }^{12}$ The authors speculated a connection between the parameters in the expression and tissue characteristics, but did not give experimental evidences or detailed analysis.

Further examinations reveal that $h_{1}^{2}$ and $h_{3}$ are also cosinoidal functions of the detection polarization angle $\theta_{s}$ [Figs. 2(b) and 2(c)]:

$$
\begin{gathered}
h_{1}^{2}=A \cos \left(4 \theta_{s}-\varphi_{1}\right)+B, \\
h_{2}=\varphi_{2}\left(\theta_{s}\right), \\
h_{3}=C \cos \left(2 \theta_{s}-\varphi_{3}\right) .
\end{gathered}
$$

Experimental data do not indicate a simple relation between $h_{2}$ and $\theta_{s}$.

Combining Eqs. (2)-(5), we obtain an analytical expression of LDP:

$$
\begin{aligned}
\operatorname{LDP}\left(\theta_{i}, \theta_{s}\right)= & \frac{1}{2} I_{\text {in }} *\left[A \cos \left(4 \theta_{s}-\varphi_{1}\right)+B\right]^{1 / 2} * \cos \left[2 \theta_{i}\right. \\
& \left.-\varphi_{2}\left(\theta_{s}\right)\right]+\frac{1}{2} I_{\text {in }} * C * \cos \left[2 \theta_{s}-\varphi_{3}\right]
\end{aligned}
$$

For the LDP image series, fitting the data at each pixel to Eq.

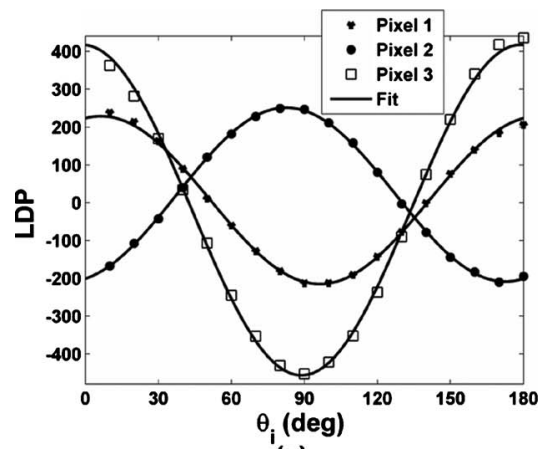

(a)

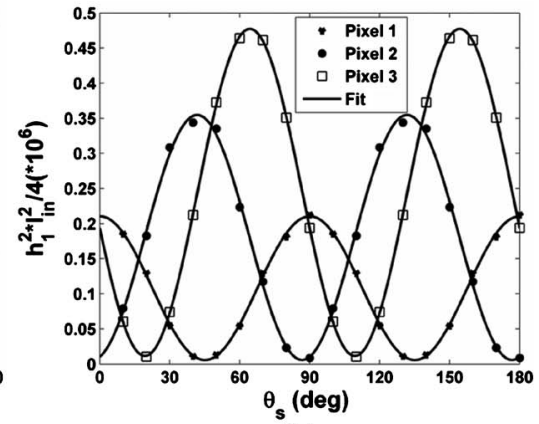

(b)

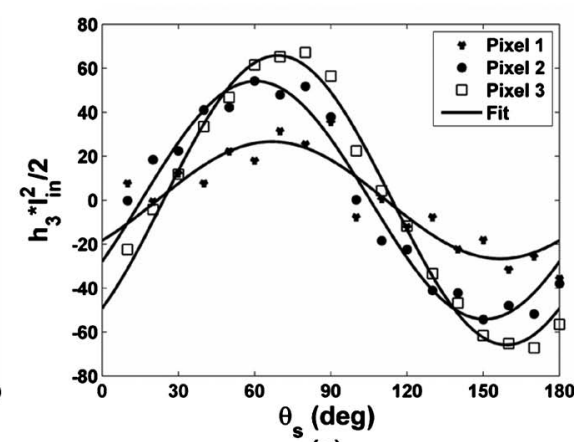

(c)

Fig. 2 Cosinoidal relationship of LDP (a) versus $\theta_{i}$ when $\theta_{\mathrm{s}}=0$, and (b) $h_{1}^{2} * l_{\text {in }}^{2} / 4$ and (c) $h_{3} * l_{\text {in }} / 2$ versus $\theta_{\mathrm{s}}$. Three curves correspond to three different pixels on the same image series of bovine skeletal muscle. Solid curves are the fitting results of the experimental data to Eqs. (2), (3), and (5). 


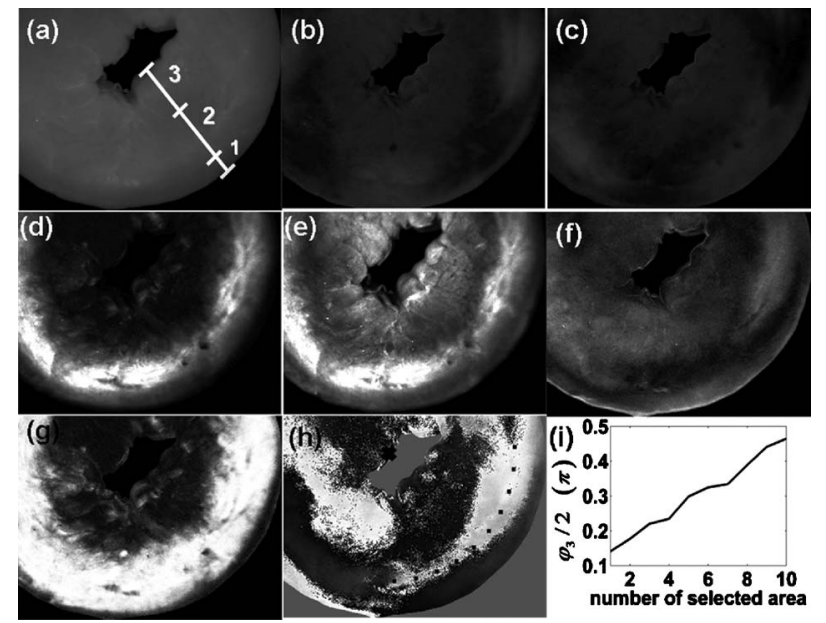

Fig. 3 Different images of the chicken heart cross section. (a): intensity image, and (b) and (c) are DOP images corresponding to incident polarization angle $\theta_{i}$ and $\theta_{i}+\pi / 4$. (d) through (h) are images of $A, B$, $C, G$, and $\varphi_{3} / 2$. (a) through (g) are normalized to the maximum value of the image. The color bar for (h) is $-\pi / 2$ to $\pi / 2$. Background is set to zero in (a) through (h). Mean values of $\varphi_{3} / 2$ are calculated within the equally spaced black squares along the arc in (h) and are shown in (i).

(6) using nonlinear least squares method results in a set of new parameters $A, B, C, \varphi_{1}$, and $\varphi_{3}$. These parameters are independent of incident and detection polarization angles and are related to different structural or optical properties of the sample. The new parameters form their own images as shown in Fig. 3. Figure 3(g) corresponds to a new parameter defined as:

$$
G \equiv A / B .
$$

More discussions about these parameters are given in following sections.

\section{Interpretation of the Fitted Parameters}

In this section, we examine in detail the parameters obtained in RLPI to understand their physical origins, particularly their relations with the structure of the biological samples.

The sample is a chicken heart (Fig. 4) whose lower part is removed to reveal a 9-mm-diam cross sectional area of the

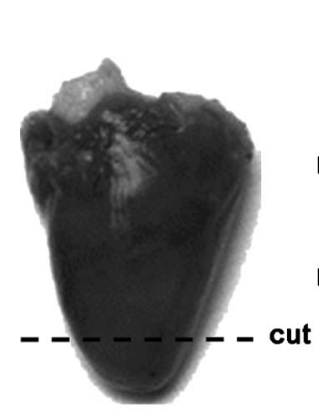

(a)

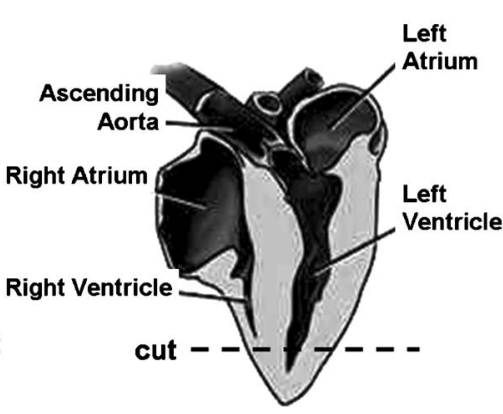

(b)
Fig. 4 (a) Picture and (b) schematic anatomical diagram of a chicken heart. The dashed line indicates the cut position.

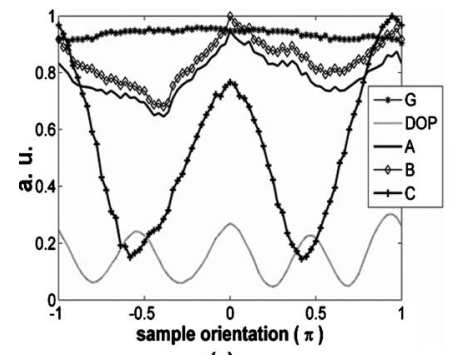

(a)

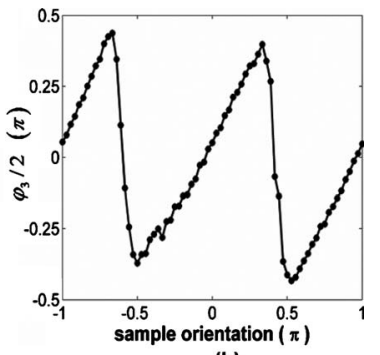

(b)
Fig. 5 Dependence of fitting parameters to sample orientation. The sample rotates around the $Z$ axis. In (a), curves for $A$ and $B$ are both normalized to the maximum of $B$, and $C$ is normalized to its own maximum.

left ventricle. Typically, 36 images are captured for each experiment, corresponding to six incident and detection polarization angles, respectively. Each experiment takes about $8 \mathrm{~s}$ limited only by the rotation mechanics of the polarizers.

Figure 3(a) shows a CCD image of the cross section. It is divided into three circular sections marked 1,2 , and 3 . It is known from anatomy that the highly fibrous heart muscles are aligned to different directions at different sections of the heart. The muscle fibers are aligned perpendicular to the imaging plane at zone 1 , and parallel to the imaging plane at zone 2 . Close to the center at zone 3, alignment of the heart muscles varies.

In the RLPI experiment, at each incident polarization angle $\theta_{i}$ we record a series of image pairs corresponding to detection polarization angles $\theta_{s}$ and $\theta_{s}+\pi / 2$. $\theta_{s}$ can take any value, but has to include the special case $\theta_{s}=\theta_{i}$. Therefore, during the RLPI experiment, we can obtain the DOP and a series of corresponding LDP different incident polarization states. Figures 3(b) and 3(c) show two DOP images of the same sample but with different incident polarization angles $\theta_{i}$ and $\theta_{i}+\pi / 4$. It is clearly demonstrated that DOP images with different incident polarization can be very different.

Figures 3(d)-3(h) show the images of $A, B, C, G$, and $\varphi_{3} / 2$. Figure $3(\mathrm{~g})$ shows clearly the three zones: $G$ is small at zone 1 and high at zone 2 . It shows more complex structures at the central area, zone 3 . Considering the anatomical structure of chicken heart, one may suspect that $G$ is higher when the fibrous structure of the sample is well aligned in the image plane. Figure 3(h) shows that in zone 2 where $G$ stays high, $\varphi_{3} / 2$ varies continuously along the circle. We choose a series of equally spaced $7 \times 7$ pixel square areas $\left(0.1 \times 0.1 \mathrm{~mm}^{2}\right.$ at the sample) along the circle, as shown in Fig. 3(h), and calculate the mean value of $\varphi_{3} / 2$. Figure 3(i) shows that $\varphi_{3} / 2$ varies almost linearly with the angle along the arc and may represent the orientation of a fibrous structure.

RLPI is independent on the incident polarization angles. Since the experimental setup is not axially symmetric, the fitted parameters may vary slightly as the sample rotates. We examine this effect by taking RLPI measurements as the sample rotates around the $\mathrm{Z}$ axis. For quantitative examination, we choose a $7 \times 7$ pixel rectangle region on the images $\left(0.1 \times 0.1 \mathrm{~mm}^{2}\right.$ at the sample) as the region of interest (ROI) and calculate the mean values of DOP, $A, B, C, \varphi_{3} / 2$, and $G$ for different sample orientations. The results are shown in Fig. 5. 
Table $1 G$ for different samples.

\begin{tabular}{|c|c|c|}
\hline Group & Sample & $G($ mean $\pm R S D)$ \\
\hline 1 & $\begin{array}{l}\text { Bovine skeletal muscle with } \\
\text { regular fiber alignment } \\
\text { parallel to sample surface }\end{array}$ & $0.91 \pm 8.8 \%$ \\
\hline 2 & $\begin{array}{l}\text { The same as group 1, but the } \\
\text { fibers are aligned perpendicular } \\
\text { to sample surface }\end{array}$ & $0.19 \pm 68 \%$ \\
\hline 3 & $\begin{array}{l}\text { Porcine skeletal muscle with } \\
\text { regular fiber alignment } \\
\text { to sample surface }\end{array}$ & $0.95 \pm 7.4 \%$ \\
\hline 4 & $\begin{array}{l}\text { Porcine skeletal muscle with } \\
\text { irregular fiber alignment to } \\
\text { sample surface }\end{array}$ & $0.63 \pm 33 \%$ \\
\hline 5 & $\begin{array}{l}\text { Gallinaceous skeletal muscle } \\
\text { with regular fiber alignment to } \\
\text { sample surface }\end{array}$ & $0.96 \pm 4.2 \%$ \\
\hline 6 & $\begin{array}{l}\text { Gallinaceous skeletal muscle } \\
\text { with irregular fiber alignment to } \\
\text { sample surface }\end{array}$ & $0.65 \pm 38 \%$ \\
\hline 7 & Porcine fatty tissue & $0.17 \pm 53 \%$ \\
\hline 8 & Porcine liver & $0.07 \pm 57 \%$ \\
\hline
\end{tabular}

Figure 5(a) shows that DOP varies with the sample orientation as a sinusoidal curve with $\pi / 2$ period. $A$ and $B$ also show periodical but nonsinusoidal variation with a period of $\pi$. The magnitude of the variation is comparable with DOP. The variation of $C$ with sample rotation is more prominent than $A$ and $B$, and the period varies at different parts of the image. $G$ stays almost constant during sample rotation, which is consistent with the assumption that $G$ is related to the order of alignment of the fibrous structure. $\varphi_{3} / 2$ varies almost linearly with the sample's rotation angle, proving that it indeed represents the orientation angle. It is also expected that the $\varphi_{3} / 2$ curve should be subject to "smoothing" as shown in Fig. 5 (b), since the orientation of the fibrous structure may not be the same within the ROI.

More evidences on the relation between $G$ and anisotropy of the sample are provided by RLPI experiments on tissues of different fibrous structure and alignment. Mean values and relative standard deviations $(\mathrm{RSD}=\mathrm{SD} /$ mean* $100 \%)$ of $G$ are calculated over $7 \times 7$ pixel ROI and listed in Table 1 .

It is clearly seen from Table 1 that there is a strong correlation between $G$ and the order of alignment, or anisotropy, of the fibrous structure in the imaging plane $(x-y$ plane). All the different skeletal muscles in groups 1,3 , and 5 contain prominent and well-aligned fibrous structures in the imaging plane. They all show very high $G$ and small RSD. Samples in group 2 are the same bovine skeletal muscle as that in group 1, but are rotated to make the well-aligned muscular fibers run perpendicular to the imaging plane. This results in a sharp reduction of anisotropy in the $x-y$ plane. Therefore, $G$ is much smaller and not well defined as the RSD increases sharply.
Samples in groups 4 and 6 are porcine and gallinaceous skeletal muscles, the same as those in group 3 and 5, but their fibrous structures look less regular. The structure is less ordered and the anisotropy should be smaller. Table 1 does show significant reduction in the measured $G$ and an increase in the RSD. Porcine liver and fat tissues in groups 7 and 8 are known to be isotropic. ${ }^{13}$ The measured $G$ s are small and the RSDs are large.

Therefore, we can conclude that $G$ and $\varphi_{3} / 2$ correlate quantitatively to the anisotropy of the fibrous structure and the fiber orientation angle in the sample. Images of these two parameters provide more objective information on the microstructure and optical property of tissues.

\section{Discussion}

Although the analytical expression for LDP, Eq. (6), was first obtained empirically, it can also be derived from the text book scattering theory for polarized photons. ${ }^{14}$ For simplicity, we consider normal incidence and detection, which do not affect the validity of the conclusion.

Stokes vector of the detected photons $S_{\text {out }}$ can be expressed as the products of the Mueller matrixes of the analyzer $M_{s}$, sample $M$, polarizer $M_{i}$, and the Stokes vector of the incident light $S_{\text {in }}$ :

$$
S_{\text {out }}=M_{s} * M * M_{i} * S_{\text {in }},
$$

where,

$$
\begin{aligned}
& M_{s}=\frac{1}{2}\left(\begin{array}{cccc}
1 & \cos 2 \theta_{s} & \sin 2 \theta_{s} & 0 \\
\cos 2 \theta_{s} & \cos ^{2} 2 \theta_{s} & \sin 2 \theta_{s} \cos 2 \theta_{s} & 0 \\
\sin 2 \theta_{s} & \sin 2 \theta_{s} \cos 2 \theta_{s} & \sin ^{2} 2 \theta_{s} & 0 \\
0 & 0 & 0 & 0
\end{array}\right) \text {, } \\
& M=\left(\begin{array}{llll}
M_{11} & M_{12} & M_{13} & M_{14} \\
M_{21} & M_{22} & M_{23} & M_{24} \\
M_{31} & M_{32} & M_{33} & M_{34} \\
M_{41} & M_{42} & M_{43} & M_{44}
\end{array}\right) \\
& M_{i}=\frac{1}{2}\left(\begin{array}{cccc}
1 & \cos 2 \theta_{i} & \sin 2 \theta_{i} & 0 \\
\cos 2 \theta_{i} & \cos ^{2} 2 \theta_{i} & \sin 2 \theta_{i} \cos 2 \theta_{i} & 0 \\
\sin 2 \theta_{i} & \sin 2 \theta_{i} \cos 2 \theta_{i} & \sin ^{2} 2 \theta_{i} & 0 \\
0 & 0 & 0 & 0
\end{array}\right) \text {, } \\
& S_{\text {out }}=\left[I_{\text {out }}, Q_{\text {out }}, U_{\text {out }}, V_{\text {out }}\right]^{T} \text {, } \\
& S_{\text {in }}=\left[I_{\text {in }}, Q_{\text {in }}, U_{\text {in }}, V_{\text {in }}\right]^{T} .
\end{aligned}
$$

If the incident light is nonpolarized, $Q_{\text {in }}=U_{\text {in }}=V_{\text {in }}=0$, we get 


$$
\begin{aligned}
\operatorname{LDP}\left(\theta_{i}, \theta_{s}\right)= & I_{\text {out }}\left(\theta_{i}, \theta_{s}\right)-I_{\text {out }}\left(\theta_{i}, \theta_{s}+\pi / 2\right) \\
= & \frac{1}{2} I_{\text {in }} *\left[A * \cos \left(4 \theta_{s}-\varphi_{1}\right)+B\right]^{1 / 2} * \cos \left[2 \theta_{i}\right. \\
& \left.-\varphi_{2}\left(\theta_{s}\right)\right]+\frac{1}{2} I_{\text {in }} * C * \cos \left(2 \theta_{s}-\varphi_{3}\right),
\end{aligned}
$$

where,

$$
\begin{gathered}
B=\left(M_{22}^{2}+M_{23}^{2}+M_{32}^{2}+M_{33}^{2}\right) / 2 ; \\
A=\left[B^{2}-\left(M_{22} M_{33}-M_{23} M_{32}\right)^{2}\right]^{1 / 2} \\
C=\sqrt{M_{21}^{2}+M_{31}^{2}} ; \\
\tan \varphi_{1}=\frac{2 M_{22} M_{32}+2 M_{23} M_{33}}{M_{22}^{2}+M_{23}^{2}-\left(M_{32}^{2}+M_{33}^{2}\right)} \\
\tan \varphi_{2}=\frac{M_{23} \cos \left(2 \theta_{s}\right)+M_{33} \sin \left(2 \theta_{s}\right)}{M_{22} \cos \left(2 \theta_{s}\right)+M_{32} \sin \left(2 \theta_{s}\right)} \\
\tan \varphi_{3}=M_{31} / M_{21} .
\end{gathered}
$$

From Eqs.(7), (15)-(17), and (20), we find that: $A, B$, and $G$ relate to the four central elements of the Mueller matrix, which couple the Stokes components $Q$ and $U$. Since both $Q$ and $U$ are defined as polarization differences, $A, B$, and $G$ should be sensitive, as in DOP, only to the structure and optical properties of the superficial layer. For $\varphi_{3} / 2$ and $C$, they are linked to Mueller matrix elements in the first column, which couple $I$ to $Q$ and $U$. Since $I$ contains contributions from all the backscattered photons, $\varphi_{3} / 2$ and $C$ should be sensitive to both superficial and deep layer properties of the sample.

More evidence on the physics origin of the RLPI parameters is also given by Monte Carlo simulations. In a spherecylinder scattering model, general anisotropic tissues are approximated to a mixture of solid spherical and infinitely long cylindrical scatterers suspended in liquid. ${ }^{15,16}$ Orientation angle of the cylindrical scatterers fluctuates around a fixed direction following a Gaussian probability distribution. The fraction of spherical scatterers and the width of the Gaussian distribution vary independently to change the anisotropy of the scattering media. Using a Monte Carlo program, ${ }^{15}$ we simulate the propagation of polarized photons in such samples and obtain the RLPI parameters following the same experimental condition and data analysis procedures as described in previous sections. For the results shown in Fig. 6, the diameters of the spherical and cylindrical scatterers are 1.5 and $2.0 \mu \mathrm{m}$, respectively, refractive indices of the scatterers and the liquid are 1.4 and 1.35 , and the total scattering coefficient is $100 \mathrm{~cm}^{-1}$.

As the fraction of spherical scatterers in the mixture or the width of the Gaussian distribution increases, the sample becomes less anisotropic. As shown in Fig. 6(a), $G$ derived from

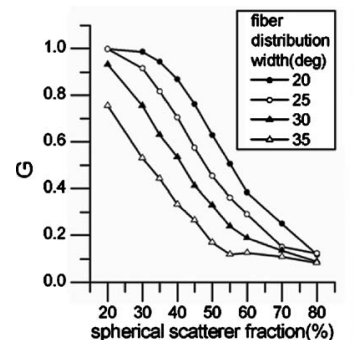

(a)

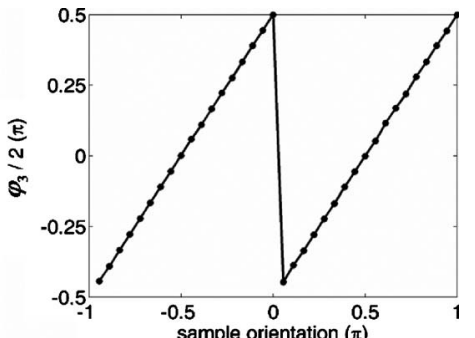

(b)
Fig. 6 (a) Dependence of $G$ on the fraction of spherical scatterers and the width of the Gaussian distribution. (b) Dependence of $\varphi_{3} / 2$ on orientation angle of the cylindrical scatterers.

the simulated data also decreases monotonously, which confirms that $G$ is related to the structural anisotropy of the sample.

Then we examine the relation between $\varphi_{3} / 2$ and the cylinder orientation. The cylindrical scatterers take $80 \%$ of the total number of scatterers and their orientation fluctuation within a Gaussian distribution of 25-deg full-width at half maxium (FWHM). As shown in Fig. 6(b), $\varphi_{3} / 2$ derived from the simulated data varies linearly with the most probable orientation of the cylinders, which confirm that $\varphi_{3} / 2$ represents the fiber orientation in the sample.

Monte Carlo simulation is also used to study the dependence of the RLPI parameters on the backscattered photons from different depths. Figure 7 shows the relation between $A$, $B, C$, and the thickness of the sample. As the thickness of the sample increases, $A$ and $B$ are quickly saturated, but $C$ keeps increasing. It is evident that backscattered photons from the superficial layer make the most significant contribution to $A$ and $B$, while photons from the much deeper layer still contribute to $C$. Therefore, both $A$ and $B$ represent the information from the superficial layer, and $C$ carries the information from deeper layers.

\section{Conclusion}

We develop a rotating linear polarization imaging (RLPI) technique. It is shown that linear polarization difference (LDP) follows an analytical relation to incident and detection

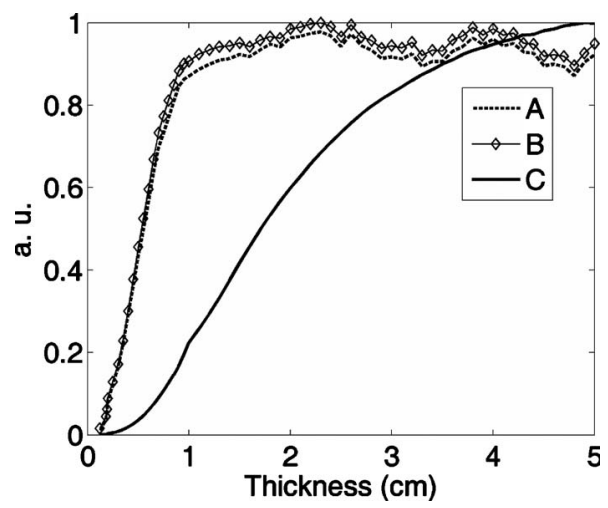

Fig. 7 Dependences of $A, B$, and $C$ on the sample's thickness. Curves for $A$ and $B$ are both normalized to the maximum of $B$, and $C$ is normalized to its own maximum. 
polarization angles. By fitting LDP images pixel by pixel to an analytical expression, we obtain a set of new images of the fitting parameters that are independent on incident polarization. Both experiments on tissues and Monte Carlo simulations prove that two of the parameters, $G$ and $\varphi_{3} / 2$, correlate to the anisotropy and orientation angle of the fibrous structure in tissues. The new technique can be used for objective and quantitative characterization of anisotropic tissues, and for clinical diagnosis of skin diseases.

\section{Acknowledgment}

This work has been supported by the National Natural Science Foundation of China (grants 60778044 and 10974114), the Ministry of Science and Technology (grant 2006CB70570), and National High Technology Research and Development Program of China (863 program number 2006AA06Z402).

\section{References}

1. S. G. Demos and R. R. Alfano, "Optical polarization imaging," Appl. Opt. 36(1), 150-155 (1997).

2. S. L. Jacques, J. R. Roman, and K. Lee, "Imaging skin pathology with polarized light," J. Biomed. Opt. 7(3), 329-340 (2002).

3. S. P. Morgan and I. M. Stockford, "Surface-reflection elimination in polarization imaging of superficial tissue," Opt. Lett. 28(2), 114-116 (2003).

4. H. R. Shao, Y. H. He, W. Li, and H. Ma, "Polarization-degree imaging contrast in turbid media: a quantitative study," Appl. Opt. 45(15), 4491-4496 (2006).

5. B. D. Cameron, M. J. Rakovic, M. Mehrubeoglu, G. W. Kattawar, S Rastegar, L. V. Wang, and G. L. Coté, "Measurement and calculation of the two-dimensional backscattering Mueller matrix of a turbid medium," Opt. Lett. 23(7), 485-487 (1998); B. D. Cameron, M. J. Rakovic, M. Mehrubeoglu, G. W. Kattawar, S. Rastegar, L. V. Wang, and G. L. Coté, "Measurement and calculation of the two- dimensional backscattering Mueller matrix of a turbid medium: errata," Opt. Lett. 23(20), 1630-487 (1998).

6. S. Manhas, M. K. Swami, P. Buddhiwant, N. Ghosh, P. K. Gupta, and K. Singh, "Mueller matrix approach for determination of optical rotation in chiral turbid media in backscattering geometry," Opt. Express 14(1), 190-202 (2006); see http://www.opticsinfobase.org/oe/ abstract.cfm?URI=OPEX-14-1-190.

7. P. J. Wu and J. T. Walsh, "Stokes polarimetry imaging of rat-tail tissue in a turbid medium: Degree of linear polarization image-maps using incident linearly polarized light," J. Biomed. Opt. 11(1), 014031 (2006).

8. T. Binzoni, C. Courvoisier, R. Giust, G. Tribillon, T. Gharbi, J. C. Hebden, T. S. Leung, J. Roux, and D. T. Delpy, "Anisotropic photon migration in human skeletal muscle," Phys. Med. Biol. 51(5), N79N90 (2006).

9. W. Cheong, S. A. Prahl, and A. J. Welch, "A review of the optical properties of biological tissues," IEEE J. Quantum Electron. 26(12), 2166-2185 (1990).

10. A. Kienle, F. K. Forster, R. Diebolder, and R. Hibst, "Light propagation in dentin: influence of microstructure on anisotropy," Phys. Med. Biol. 48(2), N7-N14 (2003).

11. A. Kienle and R. Hibst, "Light guiding in biological tissue due to scattering," Phys. Rev. Lett. 97(1), 018104 (2006).

12. S. K. Gayen, M. E. Zevallos, M. Alrubaiee, J. M. Evans, and R. R. Alfano, "Two-dimensional near-infrared transillumination imaging of biomedical media with a chromium-doped forsterite laser," Appl. Opt. 37(22), 5327-5336 (1998).

13. S. L. Jacques, J. R. Roman, and K. Lee, "Imaging superficial tissues with polarized light," Lasers Surg. Med. 26(2), 119-129 (2000).

14. C. F. Bohden and D. Huffman, "Electromagnetic theory," Chap. 2 in Absorption and Scattering of Light by Small Particles, pp 44-56, Wiley, New York (1983)

15. T. L. Yun, N. Zeng, W. Li, D. Z. Li, X. Y. Jiang, and H. Ma, "Monte Carlo simulation of polarized photon scattering in anisotropic media," Opt. Express 17(19), 16590-16602 (2009); see http:/l www.opticsinfobase.org/oe/abstract.cfm?uri =oe-17-19-16590.

16. N. Zeng, X. Y. Jiang, Q. Gao, Y. H. He, and H. Ma, "Linear polarization difference imaging and its potential applications," Appl. Opt. 48(35), 6734-6739 (2009). 